\title{
Fatores socioambientais associados à distribuição espacial de malária no assentamento Vale do Amanhecer, Município de Juruena, Estado de Mato Grosso, 2005
}

\author{
Socioenvironmental factors associated with the spatial distribution \\ of malaria in the Vale do Amanhecer settlement, \\ Municipality of Juruena, State of Mato Grosso, 2005
}

\author{
Vânia Rodrigues dos Santos ${ }^{1}$, Edna Massae Yokoo², \\ Reinaldo Souza-Santos ${ }^{3}$ e Marina Atanaka-Santos ${ }^{4}$
}

\begin{abstract}
RESUMO
Malaria é uma doença de distribuição focal. No Brasil, áreas de assentamento e garimpos de ouro na Amazônia Legal apresentam grande concentração de casos. Este artigo analisa a distribuição espacial de casos de malária, considerando fatores ambientais e sociais, no assentamento Vale do Amanhecer, Município de Juruena, Mato Grosso, Brasil. Em 2005, notificou-se 359 casos autóctones no assentamento e pelo método de Kernel identificaramse áreas de maior e menor intensidade de número de casos. As áreas de maior intensidade apresentaram 290 casos e na de menor intensidade 64 casos. A intensidade da distribuição variou no assentamento, indicando áreas de grande intensidade de casos favoráveis para transmissão como área de garimpos. Assim, apesar de assentamentos serem considerados como foco de malária, existem no seu interior, especificidades que, uma vez identificadas, podem contribuir para o controle da doença.
\end{abstract}

Palavras-chaves: Malária. Assentamento. Análise espacial. Método de Kernel.

\begin{abstract}
Malaria is a disease with focal distribution. In Brazil, settlement and gold mining areas in the Legal Amazon region present a great concentration of cases. This paper analyzes the spatial distribution of malaria cases taking into consideration environmental and social factors in the Vale do Amanhecer settlement in the municipality of Juruena, Mato Grosso, Brazil. In 2005, 359 autochthonous cases were notified in the settlement. Using the Kernel method, areas of greater and lesser intensities of case numbers were identified. The areas of greater intensity presented 290 cases and those of lesser intensity, 64 cases. The intensity of distribution varied within the settlement, thus indicating areas of great intensity of cases favoring transmission, like gold mining areas. Therefore, although the settlement was considered to be the main focus for malaria, there were specific factors within it that, once identified, could contribute towards the disease control.
\end{abstract}

Key-words: Malaria. Settlement. Spatial analysis. Kernel method.

A concentração de casos de malária, na Região Amazônica brasileira, a partir de 1975, foi relacionada à construção de rodovias, implantação de projetos agropecuários, assentamentos, mineração, garimpos e exploração de madeira que provocaram profundas modificações ambientais, ao romper o equilíbrio ecológico existente ${ }^{615}$.

\footnotetext{
1. Programa de Pós-Graduação em Saúde Coletiva, Universidade Federal de Mato Grosso, Cuiabá, MT. 2. Departamento de Epidemiologia e Bioestatística, Universidade Federal Fluminense, Niterói, RJ. 3. Departamento de Endemias Samuel Pessoa, Escola Nacional de Saúde Pública, Fundação Oswaldo Cruz, Rio de Janeiro, RJ. 4. Instituto de Saúde Coletiva, Universidade Federal de Mato Grosso, Cuiabá, MT. Apoio financeiro: PPSUS/FAPEMAT (Processo n ${ }^{0}$ 118/2005)

Endereço para correspondência: Dra Marina Atanaka-Santos. Instituto de Saúde Coletiva/UFMT. Av. Fernando Correa s/nº̣, 78060-900 Cuiabá, MT.

Fax: 556536158884

e-mail: slcs@terra.com.br

Recebido para publicação em: 03/05/2008

Aceito em: 29/01/2009
}

Em zonas de colonização ou assentamento recente, localizados nessa região $0^{1815161721}$, a transmissão da malária é atribuída à forma de ocupação do solo, exploração dos recursos naturais e circulação humana. Esses fatores que produzem grandes mudanças ambientais proporcionaram não apenas a manutenção da endemia, mas também sua dispersão. Além disso, devido à sua elevada incidência e efeitos debilitantes, provoca impacto econômico na região ao reduzir a capacidade produtiva da população acometida ${ }^{10}$.

A malária é aceita como doença predominantemente focal. No Brasil, mais especificamente na Amazônia Legal, os assentamentos e garimpos são considerados focos de maior concentração de casos.

Estudos ${ }^{116}$ na Região Amazônica, recorrendo ao uso de ferramentas de Sistemas de Informação Geográfica (SIG) tem sido realizados e demonstram que a abordagem de lugar enquanto espaço de interação entre o parasita, seres humanos e vetor 
assume relevância na compreensão de processos envolvidos na produção e reprodução da doença. No entanto, ainda são escassos estudos sobre a distribuição espacial da malária em pequenas áreas como no interior de um assentamento.

Assim, este estudo pretende analisar a distribuição espacial de casos de malária, considerando fatores ambientais e sociais no assentamento Vale do Amanhecer no Município de Juruena, Mato Grosso, em 2005.

\section{MATERIAL E MÉTODOS}

Área de estudo. 0 presente estudo foi realizado no assentamento Vale do Amanhecer, no município de Juruena, Mato Grosso, criado pelo Instituto Nacional de Colonização e Reforma Agrária (INCRA), em 1998. Localiza-se a 6,2km da sede do município, e a principal via de acesso é a MT-208, Juruena-Juara (Figura 1). Tem 14,4 mil hectares de área, no qual 7,2 mil hectares foram destinados para 0 assentamento de 250 famílias e os restantes 7,2 mil hectares foram destinados a reserva ambiental permanente.

Os lotes do assentamento têm, em média, 26 alqueires, distribuídos em estradas projetadas numeradas de 1 a 14
(Figura 1). Em 2005, residiam no assentamento Vale do Amanhecer, 718 habitantes em 200 unidades domiciliares, sendo 394 indivíduos do sexo masculino e 324 do sexo feminino.

Fonte de dados. Realizou-se um estudo descritivo e ecológico no assentamento, utilizando três fontes de dados: $1^{\text {a) }} 354$ fichas de notificação do Sistema de Informação e Vigilância Epidemiológica (SIVEP-malária) de casos de malária, fornecidas pela Secretaria Municipal de Saúde de Juruena, correspondendo a 100\% das fichas de notificação de 2005; $2^{\text {a }}$ ) levantamento domiciliar de total de residentes por domicílio segundo idade, sexo e escolaridade e $3^{\text {a }) ~ c o o r d e n a d a s ~ g e o g r a ́ f i c a s ~ d e ~} 200$ unidades domiciliares ocupadas e 24 de áreas de potenciais criadouros, garimpos e mineradora. Consideraram-se como potenciais criadouros qualquer ambiente propício ao desenvolvimento de larvas do mosquito Anopheles (igarapés, águas paradas, minas, poços, represas).

Em 2005, o município de Juruena apresentou 585 notificações de lâminas positivas para malária no SIVEP, 562 autóctones e 23 importadas. Dos casos autóctones, 359 (63,8\%) eram de moradores do assentamento dentre os quais 5 casos foram excluídos, por não identificar a localização da residência por linha (também denominada como Estrada) no assentamento. Obteve-se, assim, 354 registros de casos de malária.

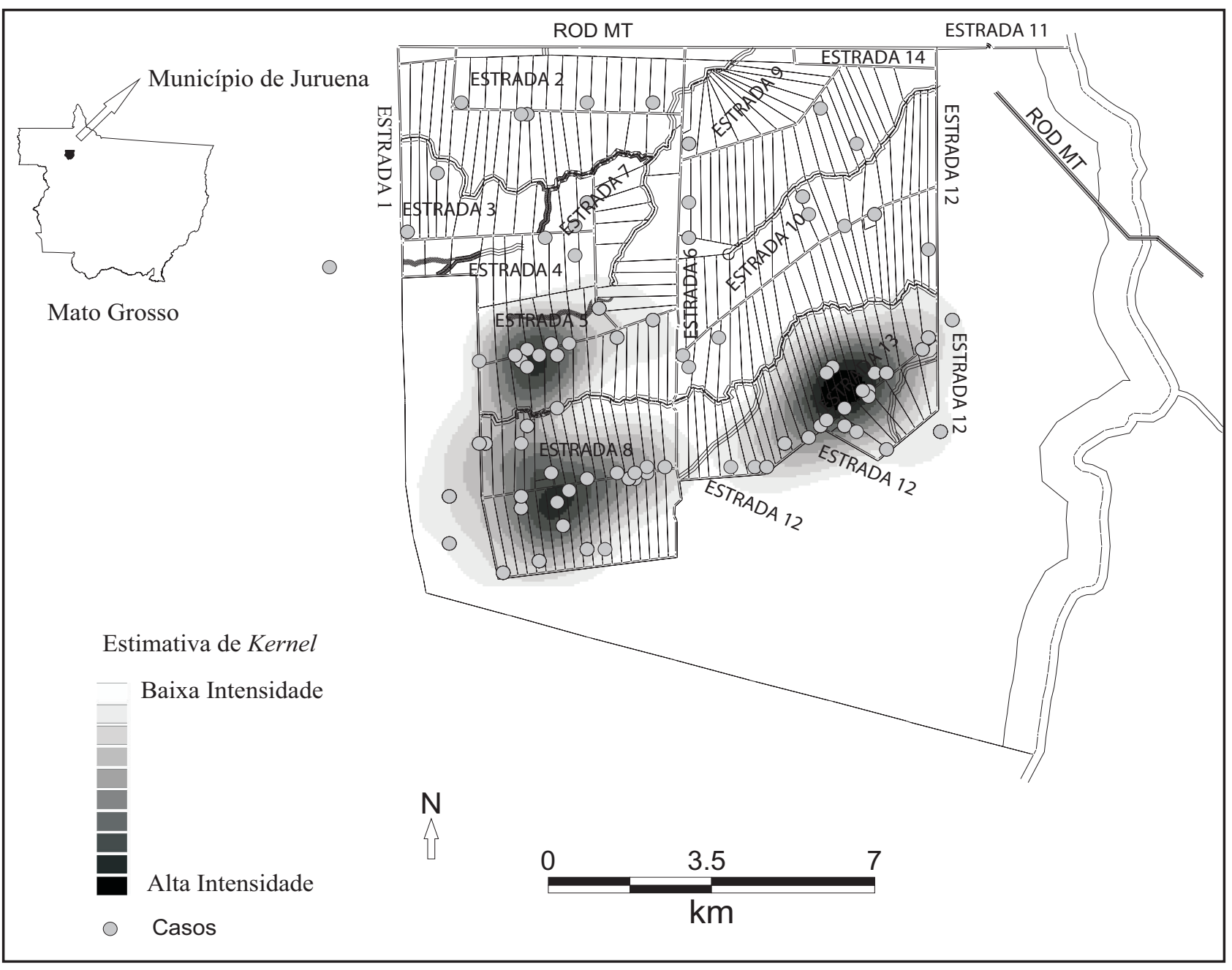

FIGURA 1

Distribuição pontual dos casos de malária em sobreposição a densidade dos casos com base na estimativa de Kernel, no assentamento Vale do Amanhecer, Juruena - MT, 2005 . 
Com o uso de GPS (Global Positioning Systems) foram aferidas as coordenadas geográficas de cada unidade domiciliar, garimpos, mineradora e potenciais criadouros. Nos potenciais criadouros coletaram-se os seguintes dados: data, horário, nome do local, tipo/especificação do criadouro e latitude e longitude.

Para a construção da base de dados, utilizou-se o software Epi Info 3.3.2 e, para a distribuição e análise espacial dos casos de malária, o software Terraview 3.1.4, ambos de livre acesso e disponibilizados nos sites www.epiinfo.com.br e www.dpi.inpe. br/terraview, respectivamente.

Análise de dados. Calculou-se a incidência parasitária anual (IPA) por 1.000 habitantes, utilizando como denominador o número de residentes por linha (estrada) e assentamento (Tabela 1), dados estes obtidos durante levantamento domiciliar e aferição das coordenadas geográficas. 0 método escolhido para analisar o padrão espacial de ocorrência da malária dos dados de distribuição pontual (coordenadas das unidades domiciliares), foi o de interpolação e alisamento pela técnica de Kernel (Equação1), que permitiu identificar áreas por intensidade de casos de malária. Utilizou-se o alisamento de Kernel para estimar a densidade de casos, com base no número de casos por unidade domiciliar.

0 uso do método denominado Kernel, considerado como método de análise exploratória de dados espaciais, além de possibilitar fácil e rápida visualização de localidades expostas, identifica diferentes graus de intensidade ${ }^{5}$ em uma determinada área. A estimativa de Kernel expressa pela Equação1, envolve uma técnica em que se coloca uma superfície simétrica sobre os pontos e, baseada em função matemática, avalia-se a distância do ponto a uma posição de referência e soma depois o valor de todas as superfícies para essa posição de referência. Esse procedimento é repetido para todas as suas posições 5 .

0 grau de alisamento foi controlado com a adoção da largura da banda (raio de influência) de $30 \mathrm{~m}$. Um raio muito pequeno poderia gerar uma superfície muito descontínua e, se fosse grande demais, a superfície poderia ficar muito suavizada. Buscou-se com esse raio, detectar padrão de distribuição dos casos.

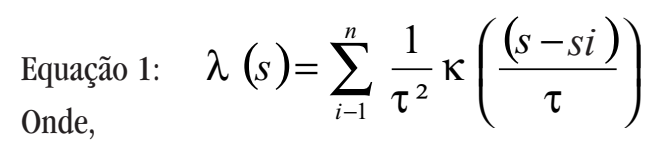

$\lambda(\mathrm{s})$ : valor estimado por área

$\tau$ : largura da banda (fator de alisamento)

$k$ ( ): função de ponderação de Kernel, no caso de uma distribuição gaussiana

$s$ : centro de cada espaço da grade regular

$s_{i}$ : local do ponto onde ocorreu o evento

Utilizou-se a denominação de baixa e alta intensidade para análise de áreas de menor e maior concentração de casos, respectivamente. Adotou-se, como área de menor intensidade, as áreas mais claras no mapa e a de maior intensidade, áreas de cores mais escuras, isto é, áreas que apresentaram mais de 80\% dos casos.

Em sistema de informações geográficas (SIG), foram calculados buffers (áreas de influência) ao redor das coordenadas das áreas de garimpo, matas e potenciais criadouros, com a finalidade de gerar áreas de exposição a possíveis fatores de risco. No cálculo dos buffers, foi utilizado raio de $400 \mathrm{~m}$, uma vez que esses fatores podem estar diretamente relacionados à dispersão do vetor.

Este estudo foi apreciado e aprovado pelo Comitê de Ética em Pesquisa do Hospital Universitário Julio Muller, Universidade Federal de Mato Grosso, sob protocolo n ${ }^{0}$ 326/CEP/HUJM/07 em 9 de maio de 2007.

\section{RESULTADOS}

A incidência parasitária anual no assentamento, em 2005, foi de 493 casos por 1.000 habitantes e a frequencia foi maior nas estradas 5, 8 e 13 concentrando $88 \%$ (315) do total de casos. Nessas estradas, a IPA foi superior a 900 casos/mil habitantes (Tabela 1). Pelo Método de Kernel, identificaram-se área de maior (290 casos) e menor (64 casos) intensidade de casos e os loteamentos localizados nas estradas 5, 8 e 13 foram as que delinearam a área de maior intensidade de casos (Figura 1).

TABELA 1

\begin{tabular}{|c|c|c|c|c|}
\hline \multirow[t]{2}{*}{ Estrada projetada (linha) } & População por & Casos & Percentagem & IPA (mil) \\
\hline & linha/estrada & $\mathrm{n}^{\mathrm{0}}$ & $\%$ & \\
\hline Estrada 2 & 104 & 04 & 1,1 & 38,5 \\
\hline Estrada 3 & 70 & 09 & 2,5 & 128,6 \\
\hline Estrada 6 & 54 & 04 & 1,1 & 74,1 \\
\hline Estrada 7 & 16 & 05 & 1,4 & 312,5 \\
\hline Estrada 9 & 89 & 06 & 1,7 & 67,4 \\
\hline Estrada 10 & 95 & 11 & 3,1 & 115,8 \\
\hline Estrada 12 & 07 & - & - & - \\
\hline Estrada 14 & 06 & - & - & - \\
\hline Estrada 5 & 64 & 58 & 16,4 & 906,2 \\
\hline Estrada 8 & 117 & 133 & 37,6 & $1.136,8$ \\
\hline Estrada 13 & 96 & 124 & 35,1 & $1.291,6$ \\
\hline Total & 718 & 354 & 100,0 & 493,0 \\
\hline
\end{tabular}

IPA: incidência parasitária anual. 
Observou-se que, ao longo de todo assentamento, existem áreas com potenciais criadouros e matas, tanto na área de maior como na de menor intensidade de casos. Já o mapa com buffer de garimpos coincide com as áreas de maior intensidade de casos, isto é, nas estradas 5,8 e 13 (Figura 2).

Os casos foram mais frequentes no sexo masculino, dos quais 48,3\% (171) encontravam-se na área de maior intensidade de casos e 9,3\% (33) na área de menor intensidade. Do total de casos na área de maior intensidade, 41,1\% (119) eram de sexo feminino, enquanto na área de menor intensidade, 48,4\% (31 casos) (Tabela 2).

0 maior número de casos ocorreu nas faixas entre 15 e 49 anos, sendo a mais atingida a de 30 a 39 anos, com 21,8\% (77) encontrados na área de maior intensidade e 4,2\% (15) na área de menor intensidade de casos. Destaca-se a ocorrência de casos em menores de 4 anos apenas na área de maior intensidade e elevado número de casos na faixa etária de 15 a 19 anos na área de menor intensidade (Tabela 2).

Quanto ao grau de instrução, observaram-se mais casos nas categorias de 1 a $3(22,6 \%)$ e 4 a $7(18,4 \%)$ anos de estudo na área de maior intensidade. Já na área de menor intensidade, 5,6\% na categoria de 1 a 3 anos de estudo, seguida de 4 a 7 anos de estudo com 4,8\% (Tabela 2).

Em relação à variável nível de parasitemia (parasitas $/ \mathrm{mm}^{3}$ de sangue), as 352 lâminas positivas registraram entre menos de 200 a 100.000 parasitas $/ \mathrm{mm}^{3}$ de sangue. Tanto na área de

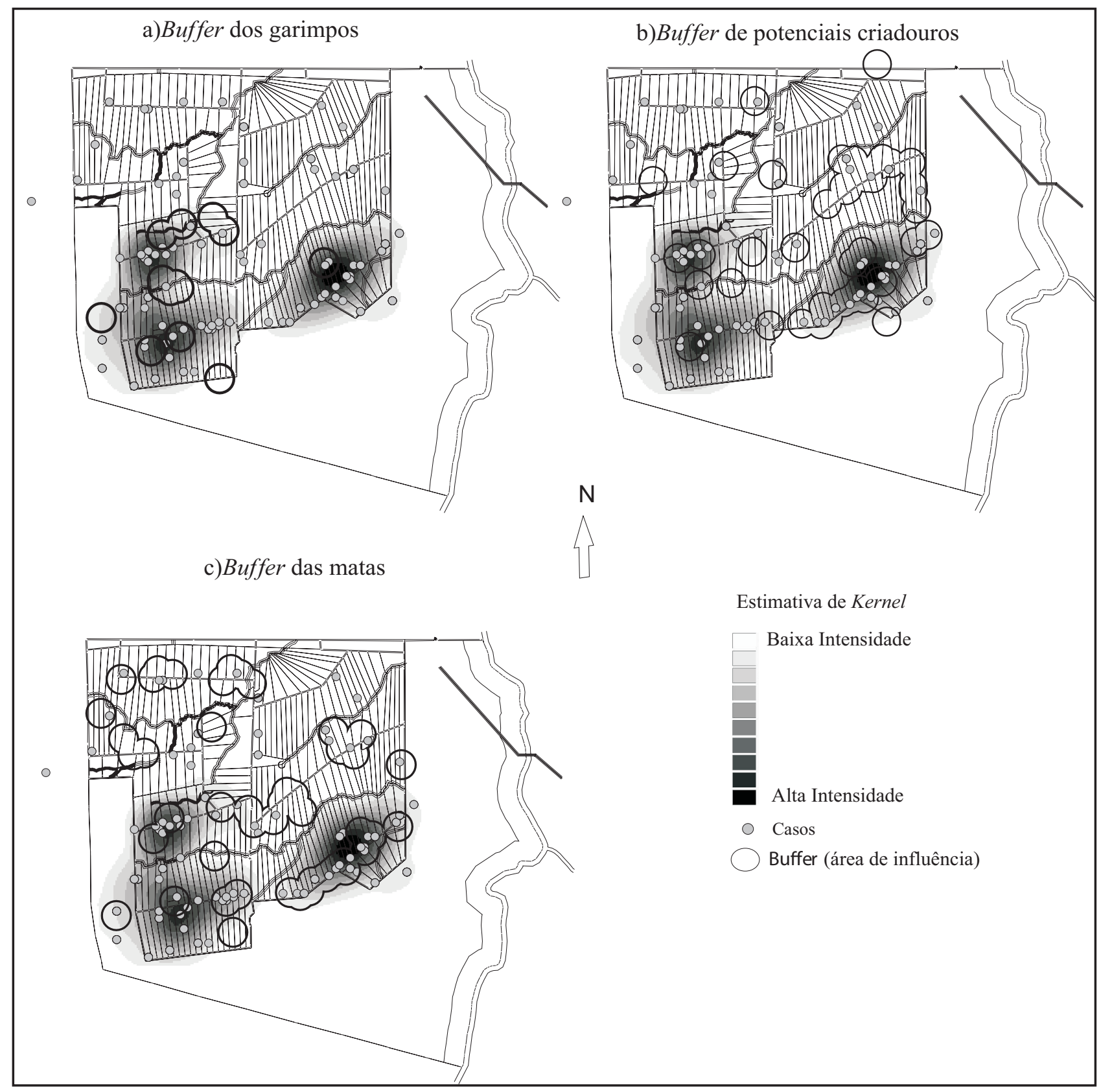

\section{FIGURA 2}

Buffers de garimpos, potenciais criadouros e matas sobrepostos ao mapa de Kernel com a distribuição pontual dos casos de malária, no assentamento Vale do Amanhecer, Juruena-MT, 2005. 
TABELA 2

Características da população com malária, segundo áreas de maior e menor intensidade de casos, no Assentamento Vale do Amanhecer, Juruena-MT, 2005.

\begin{tabular}{|c|c|c|c|c|c|c|}
\hline \multirow[t]{2}{*}{ Variável } & \multicolumn{2}{|c|}{$\begin{array}{c}\text { Área de maior } \\
\text { intensidade de casos }\end{array}$} & \multicolumn{2}{|c|}{$\begin{array}{c}\text { Área de menor } \\
\text { intensidade de casos }\end{array}$} & \multicolumn{2}{|c|}{ Total } \\
\hline & $\mathrm{n}^{0}$ & $\%$ & $\mathrm{n}^{0}$ & $\%$ & $\mathrm{n}^{0}$ & $\%$ \\
\hline \multicolumn{7}{|l|}{$\overline{\text { Sexo }}$} \\
\hline masculino & 171 & 48,3 & 33 & 9,3 & 204 & 57,6 \\
\hline feminino & 119 & 33,6 & 31 & 8,8 & 150 & 42,4 \\
\hline total & 290 & 82,0 & 64 & 18,0 & 354 & 100,0 \\
\hline \multicolumn{7}{|l|}{ Faixa etária (anos) } \\
\hline$<1$ & 5 & 1,4 & - & - & 5 & 1,4 \\
\hline $1 \mathrm{a} 4$ & 11 & 3,1 & 一 & 一 & 11 & 3,1 \\
\hline $5 \times 9$ & 22 & 6,2 & 8 & 2,3 & 30 & 8,5 \\
\hline 10 a 14 & 22 & 6,2 & 5 & 1,4 & 27 & 7,6 \\
\hline 15 a 19 & 27 & 7,6 & 13 & 3,7 & 40 & 11,3 \\
\hline 20 a 29 & 42 & 11,9 & 2 & 0,6 & 44 & 12,4 \\
\hline 30 a 39 & 77 & 21,8 & 15 & 4,2 & 92 & 26,0 \\
\hline 40 a 49 & 53 & 15,0 & 10 & 2,8 & 63 & 17,8 \\
\hline 50 a 59 & 21 & 6,0 & 6 & 1,7 & 27 & 7,6 \\
\hline 60 a 69 & 7 & 1,9 & 4 & 1,1 & 11 & 3,1 \\
\hline sem informação & 3 & 0,8 & 1 & 0,3 & 4 & 1,1 \\
\hline total & 290 & 82,0 & 64 & 18,0 & 354 & 100,0 \\
\hline \multicolumn{7}{|l|}{ Grau de instrução } \\
\hline nenhum & 46 & 13,1 & 14 & 4,0 & 60 & 17,0 \\
\hline 1 a 3 & 80 & 22,7 & 20 & 5,7 & 100 & 28,4 \\
\hline $4 \mathrm{a} 7$ & 65 & 18,5 & 17 & 4,8 & 82 & 23,3 \\
\hline 8 a 11 & 16 & 4,5 & 3 & 0,9 & 19 & 5,4 \\
\hline$\geq 12$ & 1 & 0,3 & - & & 1 & 0,3 \\
\hline não se aplica & 22 & 6,3 & 2 & 0,6 & 24 & 6,8 \\
\hline sem informação & 58 & 16,5 & 8 & 2,3 & 66 & 18,8 \\
\hline total* & 288 & 81,8 & 64 & 18,2 & 352 & 100,0 \\
\hline \multicolumn{7}{|c|}{ Parasitemia** (parasitas por $\mathrm{mm}^{3}$ de sangue) } \\
\hline$<200$ & 37 & 10,5 & 4 & 1,1 & 41 & 11,6 \\
\hline $200-300$ & 68 & 19,3 & 8 & 2,3 & 76 & 21,6 \\
\hline $301-500$ & 51 & 14,5 & 13 & 3,7 & 64 & 18,2 \\
\hline $501-10.000$ & 119 & 33,8 & 37 & 10,5 & 156 & 44,3 \\
\hline $10.001-100.000$ & 13 & 3,7 & 2 & 0,6 & 15 & 4,3 \\
\hline total* $^{*}$ & 288 & 81,8 & 64 & 18,2 & 352 & 100,0 \\
\hline \multicolumn{7}{|l|}{ Resultado de exame } \\
\hline malaria falciparum & 121 & 34,4 & 20 & 5,7 & 141 & 40,1 \\
\hline malaria vivax & 166 & 47,1 & 44 & 12,6 & 210 & 59,7 \\
\hline malaria Mista & 1 & 0,3 & - & - & 1 & 0,3 \\
\hline $\operatorname{total}^{*}$ & 288 & 81,8 & 64 & 18,9 & 352 & 100,0 \\
\hline \multicolumn{7}{|l|}{ Sintomas (horas) } \\
\hline sem informação & 92 & 26,0 & 24 & 6,8 & 116 & 32,8 \\
\hline$\leq 24$ & 78 & 22,0 & 14 & 4,0 & 92 & 26,0 \\
\hline 25 a 48 & 43 & 12,1 & 13 & 3,7 & 56 & 16,0 \\
\hline 49 a 72 & 27 & 7,6 & 8 & 2,3 & 35 & 9,8 \\
\hline$\geq 73$ & 50 & 14,1 & 5 & 1,4 & 55 & 15,5 \\
\hline total & 290 & 82,0 & 64 & 18,0 & 354 & 100,0 \\
\hline
\end{tabular}

*2 fichas de notificação (considerados casos) não apresentaram preenchimento no campo de Grau de Instrução, Parasitemia e Espécie parasitária, não sendo incluídas nesta tabela. ***não houve notificação de casos com parasitemia superior a 100.000 parasitas por $\mathrm{mm}^{3}$ de sangue. 
maior como na de menor intensidade, predominaram lâminas entre 501 a 10.000 parasitos por $\mathrm{mm}^{3}$ de sangue. No entanto, destaca-se a ocorrência de 3,7\% (13) e 0,6\% (2) de casos com parasitemia de 10.001 a 100.000 parasitos $/ \mathrm{mm}^{3}$ de sangue em áreas de maior e menor intensidade respectivamente.

A infecção por Plasmodium vivax foi maior na área de maior concentração de casos 47,1\% (166), seguida pelo Plasmodium falciparum 34,4\% (121). 0 mesmo ocorreu na área de menor intensidade de casos em que a espécie parasitária predominante era Plasmodium vivax com 12,6\% (44), seguida pelo Plasmodium falciparum com 5,7\% (20) (Tabela 2).

Em relação à diferença (em horas) entre o início dos sintomas e o início do tratamento, não há informações em 26\% (92) das notificações na área de maior intensidade e 6,8\% (24) na área de menor intensidade. Assim, do total de 198 casos com o registro início dos sintomas e o início do tratamento na área de maior intensidade de casos, 39,4\% (78) iniciou o tratamento antes ou em 24 horas após o surgimento dos primeiros sintomas e 38,9\% (77) no intervalo superior a 48 horas. Do total de 40 casos na área de menor intensidade, o intervalo entre o início dos sintomas e 0 início do tratamento, foi menor ou igual a 24 horas em 35\% (14) dos casos e 32,5\% (13) superior a 48 horas (Tabela 2).

\section{DISCUSSÃO}

A ocorrência da malária é determinada por uma cadeia epidemiológica e para que esta se complete é necessário que um conjunto de fatores primordiais: o agente etiológico (protozoário), o vetor (mosquito Anopheles), o hospedeiro intermediário suscetível (homem) e o ambiente propício para a proliferação mosquito.

No assentamento Vale do Amanhecer, a malária apresentou tendência ascendente com 44 (IPA:7,5 casos/mil habitantes), 248 (IPA:40 casos/mil habitantes) e 728 (IPA:116,8 casos/mil habitantes) casos, nos anos de 2002, 2003 e 2004, respectivamente 9 No ano de 2005, houve um incremento maior alcançando a IPA de 493 casos/mil habitantes, principalmente concentrada em áreas próximas aos garimpos. Esse incremento de casos, de 2003 a 2005 , foi atribuído à instalação de garimpos no assentamento.

Diversos autores como Atanaka-Santos ${ }^{1}$, Barbieri ${ }^{3}$, Barbieri \& Sawyer ${ }^{4}$, Paula $^{14}$, Ribeiro ${ }^{15}$, Rodrigues ${ }^{16}$, Salcedo ${ }^{17}$, Sawyer ${ }^{18}$, Spencer ${ }^{19}$, relacionam o surgimento de casos de malária à construção de rodovias, à implantação de projetos agropecuários, à assentamentos, aos desmatamentos, à mineração, aos garimpos e à exploração de madeira que provocaram profundas modificações ambientais ao romper o equilíbrio ecológico existente.

Além disso, diversos estudos mostram que a distribuição da malária não ocorre de forma homogênea entre as diversas regiões do país, verificando-se que mesmo dentro de um estado, a distribuição é desigual ${ }^{12713}$. Tadei ${ }^{20}$ verificou, através de inquéritos entomológicos na região amazônica, a existência de diferenças marcantes quanto à densidade de anofelinos, não só entre as diferentes áreas estudadas como também dentro de uma mesma área. Segundo Gaudart cols ${ }^{11}$, em estudo realizado na aldeia de Mali na África, embora toda a região seja classificada como zona de alto risco para a malária, a identificação de clusters demonstrou a alta variabilidade do risco da malária no tempo e no espaço.

Os resultados desta pesquisa mostram que, mesmo em uma área geográfica menor como assentamento Vale do Amanhecer com 7,2 mil hectares, com características aparentemente semelhantes, a distribuição é heterogênea, existindo maior concentração de casos em algumas áreas do que em outras. o mapa de Kernel permitiu visualizar e circunscrever área de maior intensidade de casos e, o buffer dos garimpos confirmou a localização dos mesmos nessa área.

Dos 354 casos notificados, 290 encontravam-se na área de maior intensidade de casos, onde há combinação de fatores como garimpo, mata e potenciais criadouros. Apenas 64 casos encontravam-se fora da área de maior intensidade de casos, isto é, distribuídos por todo assentamento, possivelmente sob infuência direta da proximidade dos potenciais criadouros e matas.

Segundo Barbieri ${ }^{4}$ e Guimarães ${ }^{12}$, as maiores prevalências de malária na Região Norte Matogrossense ocorrem em áreas garimpeiras ou áreas rurais e urbanas próximas a garimpos. Em áreas de garimpos há grande quantidade de locais propícios à proliferação dos vetores como acúmulo de água em cavas abandonadas. Além do ambiente físico favorável a reprodução de vetores, há também outros fatores como: contato mais próximo do homem com o vetor devido à exposição constante (trabalho no horário de ação dos vetores); qualidade precária das moradias que além de não possuírem paredes são cobertas de palha ou plástico preto, que oferecem pouca proteção contra a ação dos vetores e; proximidade dos barracões (moradias) com locais de trabalho (cavas).

As características dos casos, na área de maior intensidade não diferem muito dos casos na área de menor intensidade; as duas áreas possuem maior número de indivíduos do sexo masculino que do feminino, maior freqüiência na faixa etária de 30 a 39 anos e escolaridade menor de 4 anos. No entanto, a ocorrência de casos em menores de 4 anos somente na área de maior intensidade de casos indica o risco de transmissão domiciliar, levando a pressupor a precariedade das moradias e exposição aos riscos relacionados aos garimpos localizados nessa área.

Do total de 198 casos, com registro sobre início de sintomas e início de tratamento, 37,8\% (90) dos casos apresentaram intervalo superior a 48 horas. A demora no início de tratamento amplia o risco de evolução da doença em sua forma mais grave e incrementa o potencial de transmissão pela circulação da forma infectante do parasito no sangue. Assim, a presença e circulação do ser humano infectado e do vetor, aliado às condições ambientais propícias (matas dos lotes e reserva, coleções de água, riachos e moradias precárias) e ao desequilíbrio ecológico provocados pelos garimpos no assentamento, este último localizado na área de maior intensidade de casos, favorecem a instalação e manutenção de malária, considerando a existência dos vetores e pessoas infectadas e suscetíveis na região.

A adoção do assentamento como unidade de análise ressaltou diferentes intensidades na distribuição de casos no assentamento, estando em geral relacionada com a forma de uso/ocupação do 
solo e a consequiente degradação ambiental. A análise espacial adotada permitiu esclarecer a heterogeneidade da distribuição de malária em pequenas áreas e a análise em nível local - de famílias e domicílios -, utilizando a técnica de SIG, tornou possível identificar o padrão dessa heterogeneidade de risco de transmissão.

A heterogeneidade da distribuição da malária encontrada no assentamento Vale do Amanhecer reforça a necessidade de revisão da dimensão espacial envolvendo a concepção de distribuição focal da malária na Região Amazônica e análises mais aprofundadas sobre os fatores que influenciam a distribuição dos casos, especialmente em pequenas áreas.

\section{REFERÊNCIAS}

1. Atanaka-Santos M, Czeresnia D, Souza-Santos R, Oliveira RM. Comportamento epidemiológico da malária no Estado de Mato Grosso, 1980-2003. Revista da Sociedade Brasileira de Medicina Tropical 39: 187-192, 2006.

2. Barata RB. Malária e seu controle. Editora Hucitec, São Paulo, 1998.

3. Barbieri AF. Uso antrópico da terra e malária no norte de Mato Grosso, 1992 a 1995. Dissertação de Mestrado. Universidade Federal de Minas Gerais, Belo Horizonte, MG, 2000.

4. Barbieri AF, Sawyer DO. Heterogeneidade da prevalência de malária em garimpos do norte de Mato Grosso, Brasil. Cadernos de Saúde Pública 23: 2878-2886,2007.

5. Câmara G, Carvalho MS. Análise espacial de eventos. In: Druck S, Carvalho MS, Câmara G, Monteiro AMV (eds) Análise espacial de dados geográficos. Empresa Brasileira de Pesquisa Agropecuária Cerrados (Embrapa Cerrados), Planaltina, Distrito Federal, p.53-122, 2004.

6. Carter R, Mendis KN, Roberts D. Spatial targeting of inteventions against malaria. Bulletin of the World Health Organization 78: 1401-1411, 2000.

7. Castilla REF, Sawyer DO. Malaria rates and fate: a socioeconomic study of malaria in Brazil. Social Science and Medicine 37: 1137-1145, 1993.

8. Cordeiro CES, Filomeno CRM, Costa CMA, Couto AARA. Perfil epidemiológico da malária no Estado do Pará, em 1999, com base numa série histórica de dez anos (1980-1999). Informe Epidemiológico do Sistema Único de Saúde 11: 69-77, 2002.

9. Ferreira IM. Fatores associados à ocorrência da malária em área de assentamento, município de Juruena - MT. Dissertação de mestrado. Universidade Federal de
Mato Grosso, Cuiabá, MT, 2007.

10. Fundação Nacional de Saúde. Guia de Vigilância Epidemiológica. $5^{a}$ edição, Secretaria de Vigilância em Saúde Ministério da Saúde, Brasília, 2002.

11. Gaudart J, Poduiougou B, Dicko A, Ranque S, Toure O, Sagara I, Diallo M, Diawara S, Outarra A, Diakite M, Duombo OK. Space-time clustering of childhood malaria at the household leve: a dynamic cohort in Mali village. BioMed Central Public Health 6: 286, 2006.

12. Guimarães AE, Gentile C, Alencar J, Lopes CM, Mello RP. Ecology of Anopheline (Diptera, Culicidae), malaria vectors around the Serra da Mesa reservoir, state of Goiás, Brazil. 1 Frequency and climatic factors. Cadernos de Saúde Pública 20: 291-302, 2004.

13. Oliveira Junior JG. Análise da distribuição da malária na Amazônia brasileira, enfoque municipal. Dissertação de Mestrado. Instituto de Medicina Social da Universidade do Estado do Rio de Janeiro, Rio de Janeiro, 2001.

14. Paula JA, Brito FRA, Amaro JJV, Nabuco MR. Fundamentos históricos e metodológicos da questão ambiental. In: Paula JA (coord) Biodiversidade, população e economia: uma região de Mata Atlântica. Universidade Federal de Minas Gerais/ Centro de Desenvolvimento e Planejamento Regional, Belo Horizonte, cap. 5, p. 201-255, 1997.

15. Ribeiro MCT, Goncalves EGR, Tauil PL, Silva AR. Aspectos epidemiológicos de um foco de malária no município de São Luis, Maranhão, 2003. Revista da Sociedade Brasileira de Medicina Tropical 38: 272-274, 2005.

16. Rodrigues AF, Escobar AL, Souza-Santos R. Análise espacial e determinação de áreas para o controle da malária no Estado de Rondônia. Revista da Sociedade Brasileira de Medicina Tropical 41: 55-64, 2008.

17. Salcedo JMV, Camargo EP, Krieger H, Silva LHP, Camargo LMA. Malaria control in an agro-industrial settlement of Rondônia (Western Amazon Region, Brazil). Memórias do Instituto Oswaldo Cruz 95: 139-145, 2000.

18. Sawyer DR. População e meio ambiente na Amazônia brasileira. In: Martine G (org) População, meio ambiente e desenvolvimento sustentável: verdades e contradições. $2^{a}$ edição. Editora Universidade de Campinas, Campinas, p.149-170, 1996

19. Spencer BR. Gold mining and malaria in the Brazilian Amazon. Dissertação mestrado. Departament of Epidemiology and Public Health, Yale University, New Haven, 1996.

20. Tadei WP, Santos JMM, Costa WLD S, Scarpassa, VM. Biologia dos Anofelinos Amazônicos. Ocorrência de Espécies deAnopheles, Dinâmica da Transmissão e Controle da Malária na Zona Urbana de Ariquemes (Rondônia). Revista Instituto de Medicina Tropical. São Paulo 30: 221-251, 1988.

21. Vasconcelos CH, Novo EMLM, Donalisio MR. Uso do sensoriamento remoto para estudar a influência de alterações ambientais na distribuição da malária 